\title{
Ablação com Radiofreqüência do Flutter Atrial Tipo I. Importância do Bloqueio Bidirecional do Istmo entre a Veia Cava Inferior e o Anel da Valva Tricúspide
}

\author{
Mauricio Scanavacca, Eduardo Sosa, José Luis Velarde, André D’Ávila, Denise Hachul, \\ Basileu Reolão, Osvaldo Sanches, Márcio Silva, Francisco Darrieux
}

São Paulo, SP

\begin{abstract}
Objetivo - Estudar a importância clínica da determinação eletrofisiológica da presença de bloqueio bidirecional na condução pelo istmo localizado entre a veia cava inferior e o anel da valva tricúspide (VCI $A T)$, após a ablação do flutter atrial tipo I (FL) com radiofreqüencia $(R F)$.

Métodos - Quarenta pacientes consecutivos (idade média $51 \pm 11$ anos) com FL foram submetidos a ablação do istmo VCI-AT com RF. Em 30 pacientes (GI), o sucesso foi avaliado pela interrupção e não reindução do FL com estimulação atrial programada. Nos últimos 10 pacientes foi avaliada também a condução bidirecional pelo istmo, com cateteres posicionados na sua entrada e saída e em cada lado da linha de bloqueio. O bloqueio foi considerado bidirecional quando ocorrido nos dois sentidos e unidirecional quando ocorrido em um só sentido.

Resultados - Vinte e seis (86\%) pacientes do GI e $10(100 \%)$ do GII tiveram sucesso imediato ( $p=$ 0,5558). Durante o seguimento, 7 (30\%) de 23 pacientes do GI e 3 (30\%) de 9 do GII tiveram recorrência de $F L(p=N S)$. Os três pacientes do GII que apresentaram recorrência tinham bloqueio unidirecional, enquanto os seis casos sem recorrência tinham bloqueio bidirecional $(p=0,012)$.

Conclusão - A demonstração de bloqueio bidirecional no istmo VCI-AT, obtida imediatamente após a ablação do FL com RF, relaciona-se a menor índice de recorrência clínica, devendo ser o critério preferencial para término do procedimento.
\end{abstract}

Palavras-chave: flutter atrial, ablação com radiofreqüência, taquicardias supraventriculares

\section{Type I Atrial Flutter Radiofrequency Ablation. Importance of Bidirectional Line of Block in the Inferior Vena Cava Tricuspide Annulus Isthmus}

\begin{abstract}
Purpose - To determine the clinical importance of a bi-directional line of block demonstration in the inferior vena cava-tricuspid annulus isthmus as an end-point for radiofrequency $(R F)$ atrial flutter $(F L)$ ablation.

Methods - Forty consecutive patients (51 11 years) with type I FL were divided in 2 groups: GI (30 patients) anatomic, non-electrophysiologic isthmus ablation technique (interruption and non-induction FL criteria); and GII (10 patients) anatomic with electrophysiologic evaluation of bi-directional isthmus conduction. The isthmus activation was analyzed before and after anatomic RF ablation with a cateter exploring each side of the line of block, depending on the conduction evaluation (anterograde or retrograde).

Results - FL was interrupted and not reinduced in 26/ $30(86.6 \%)$ GI patients and in $10(100 \%)$ GII patients ( $p=$ 0.5558). During follow-up FL recurred in $30 \%$ of the patients in both groups. In GII, 6 patients with bi-directional block remained assymptomatic, whereas 3 patients with unidirectional block presented recurrence $(p=0.012)$.

Conclusion - Electrophysiologic demonstration of bidirectional line of block in the isthmus is related to longterm success and should be the criterion for interruption of type I atrial FL RF ablation.
\end{abstract}

Key-words: atrial flutter, radiofrequency catheter ablation, supraventricular tachycardia
Instituto do Coração do Hospital das Clínicas - FMUSP

Correspondência: Mauricio I. Scanavacca - InCor - Divisão Clínica - Av. Dr. Enéas C. Aguiar, 44 - 05403-000 - São Paulo, SP

Recebido para publicação em 31/10/97

Aceito em 20/8/98
A ablação com radiofreqüência (RF) doflutter atrial do tipo I vem apresentando resultados promissores desde $1992^{1-7}$. As técnicas aplicadas basearam-se em demonstrações eletrofisiológicas de que seu circuito é macrorreen- 
trante, restrito ao átrio direito, e utiliza necessariamente um istmo de tecido atrial localizado entre a veia cava inferior (VCI) e o anel da valva tricúspide (AT) ${ }^{8-12}$.

As aplicações de RF têm como objetivo provocar uma linha de bloqueio entre a VCI e o AT inviabilizando a sustentação do circuito do flutter. Supunha-se que esse bloqueio ocorresse quando houvesse interrupção do flutter durante aplicação de RF, não reindutível com estimulação atrial programada e que esses dois sinais fossem utilizados como critérios para estabelecer eficácia e término do procedimento ${ }^{1-7}$.

Embora a taxa de interrupção do flutter fosse alta, os resultados clínicos a longo prazo mostraram alta taxa de recorrência $^{1-7}$. Duas hipóteses foram formuladas para explicar essas recorrências: 1) recuperação da condução durante o seguimento tardio; 2) realização de bloqueio incompleto na condução pelo istmo VCI-AT, suficiente para interromper o flutter, mas insuficiente para impedir sua recorrência tardia.

Entre 1995 e 1996, surgiram várias evidências de que a segunda hipótese é o fator mais importante para as recorrências clínicas ${ }^{13-17}$. Esses estudos sugeriram que a demonstração eletrofisiológica de bloqueio bidirecional na condução pelo istmo VCI-AT, após aplicações de RF, relaciona-se com menor recorrência durante seguimento clínico. Os critérios da presença de bloqueio uni e bidirecional na condução pelo istmo basearam-se na mudança da seqüência da ativação atrial em torno do AT durante estimulação atrial, obtida por cateter com 10 pares de eletrodos ${ }^{13-17}$.

Opresente estudo foi desenvolvido, explorando o mesmo conceito com técnica distinta. Nosso objetivo foi desenvolver uma técnica de avaliação da condução pelo istmo VCI-AT, determinar a relevância da demonstração do bloqueio da condução pelo istmo, como critério para o término do procedimento na redução das recorrências de flutter e analisar os resultados clínicos tardios da ablação com RF doflutter do tipo I.

\section{Métodos}

Entre novembro/93 e junho/96, 40 pacientes consecutivos portadores de flutter do tipo I (ondas P negativas em DII, DIII e aVF e freqüência atrial maior de 240bpm) foram submetidos à ablação seletiva com RF do istmo VCI-AT.

As características clínicas dos pacientes encontramse na tabela I. Todos apresentavam palpitações taquicárdicas recorrentes ou manifestações clínicas de insuficiência

Tabela I - Estatísticas descritivas das características clínicas dos pacientes submetidos à ablação de flutter atrial tipo I com radiofreqüência

$\begin{array}{ll}\text { Idade média (anos) } & 51 \pm 11 \\ \begin{array}{l}\text { Sexo (masculino/feminino) } \\ \text { Cardiopatias associadas }\end{array} & 35 / 5 \\ \text { Ausente } & 26(65 \%) \\ \text { Congênita operada } & 7(17,5 \%) \\ \text { Cardiomiopatia dilatada idiopática } & 3(7,5 \%) \\ \text { Cardiopatia chagásica } & 1(2,5 \%) \\ \text { Cardiopatia isquêmica } & 1(2,5 \%) \\ \text { Hipertensão arterial sistêmica } & 1(2,5 \%) \\ \text { Displasia de ventrículo direito } & 1(2,5 \%)\end{array}$

cardíaca secundária à freqüência cardíaca elevada e haviam sido submetidos a pelo menos uma cardioversão elétrica, apesar do uso de drogas antiarrítmicas. Os pacientes com algum episódio de fibrilação atrial (FA), documentado previamente ao procedimento, foram excluídos.

Todos os pacientes foram submetidos à ablação do istmo VCI-AT, conforme descrição prévia ${ }^{6}$, segundo a téc-

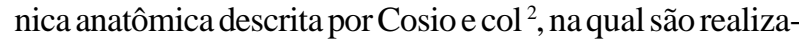
das aplicações subseqüentes de RF do AT à borda da VCI, com objetivo de realizar uma linha de bloqueio à condução do estímulo elétrico entre essas estruturas. De acordo com o critério de avaliação utilizado no final do procedimento, os pacientes foram divididos em dois grupos: GI (30 pacientes iniciais): interrupção do flutter durante aplicação de RF e não reindução pela estimulação atrial programada (antes e após infusão de isoproterenol); GII (10 pacientes finais): mesmo método anterior com posterior avaliação eletrofisiológica da condução bidirecional pelo istmo VCI-AT.

O método de avaliação da condução pelo istmo VCIAT, realizado nos pacientes do GII, baseou-se em duas hipóteses: I) um estímulo aplicado na entrada do istmo VCIAT, durante ritmo sinusal, atinge o óstio do seio coronário (saída do istmo), seguindo três caminhos possíveis: 1) curto - através do istmo VCI-AT; 2) intermediário - contornando a VCI; 3) longo - contornando o AT. Um estímulo aplicado no óstio do seio coronário deve seguir os mesmos caminhos, no sentido contrário, para atingir a entrada do istmo VCI-AT (fig. 1); II) Uma vez realizado o bloqueio, um estímulo aplica-

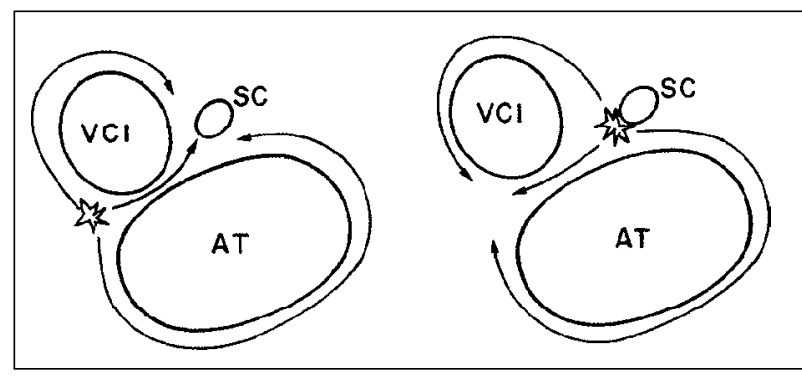

Fig. 1 - Hipótese I: para avaliação da condução pelo istmo veia cava inferior (VCI) anel da valva tricúspide (AT); SC- óstio do seio coronário. * artefato do estímulo elétrico. As setas apontam os possíveis caminhos de propagação do estímulo aplicado na entrada e saída do istmo VCI-AT.

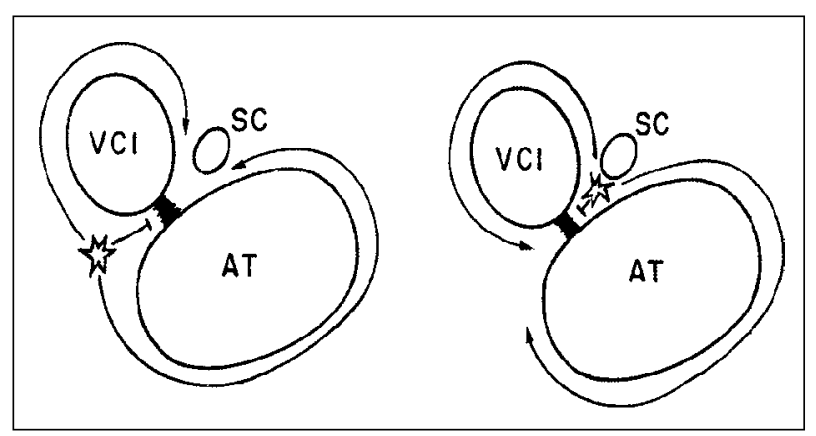

Fig. 2 - Hipótese II: após a realização do bloqueio na condução anterógrada pelo istmo veia cava inferior (VCI) - anel da valva tricúspide (AT), um impulso elétrico aplicado na entrada propaga-se pelo átrio direito contornando a VCI e o AT para atingir o seio coronário. Após o bloqueio retrógrado na condução pelo istmo VCI-AT, o estímulo segue o mesmo caminho, no sentido inverso. 
do na entrada do istmo VCI-AT deve, necessariamente, contornar a VCI e o AT para atingir o óstio do seio coronário (fig. 2). Portanto, a saída do istmo VCI-AT deve ser ativada, retrogradamente, por esta onda de ativação. O estímulo atrial aplicado nas vizinhanças do óstio do seio coronário segue a mesma direção para atingir a entrada do istmo VCIAT, no sentido contrário.

Para realizar esta demonstração foram utilizados quatro cateteres, que registraram os eletrogramas atriais em quatro áreas específicas em torno da VCI. Um $1^{\circ}$ cateter, quadripolar ou octacapolar, posicionado no átrio direito lateral com o eletrodo distal próximo à entrada do istmo (CT 1=entrada do istmo); um $2^{\circ}$, deflectível, com ponta de 4 ou $8 \mathrm{~mm}$, posicionado noistmo AT-VCI(CT2=istmo medial); um $3^{\circ}$, quadripolar, introduzido no seio coronário, deixando o par proximal posicionado no óstio (CT 3= saída do istmo) e um $4^{\circ}$, deflectível, posicionado na borda superior da VCI(CT4) (fig. 3).

Estando o paciente em ritmo sinusal foi realizada estimulação atrial contínua nos ciclos de $600 \mathrm{~ms}$ ou $500 \mathrm{~ms}$, através dos cateteres 1 (entrada do istmo) e 3 (saída do ist- mo) e a seqüência de ativação dos eletrogramas foi estudada antes e após as aplicações de RF no istmo. Os critérios de indução de bloqueio da condução no istmo foram: a) bloqueio anterógrado: sequiência de ativação atrial CT-1; CT-4; CT-3; CT-2 durante estimulação no cateter-1; b) bloqueio retrógrado: sequiência de ativação atrial CT-3; CT-4; CT-1; CT-2 durante estimulação no cateter-3 (fig. 4). Considerouse bloqueio bidirecional na condução pelo istmo VCI-AT, quando os critérios de bloqueio anterógrado e retrógrado foram atingidos. O bloqueio unidirecional foi definido quando o bloqueio na condução pelo istmo ocorreu isoladamente em um sentido.

Os pacientes com sucesso na ablação do flutter receberam alta sem drogas antiarrítmicas e foram submetidos à avaliação clínica em um mês e, em seguida, a cada três meses. Holter e estudo eletrofisiológico foram realizados, quando necessários, para esclarecer palpitações não documentadas ao eletrocardiograma convencional.

A análise estatística consistiu na comparação das médias das variáveis idade, ciclo doflutter (em ms), número de

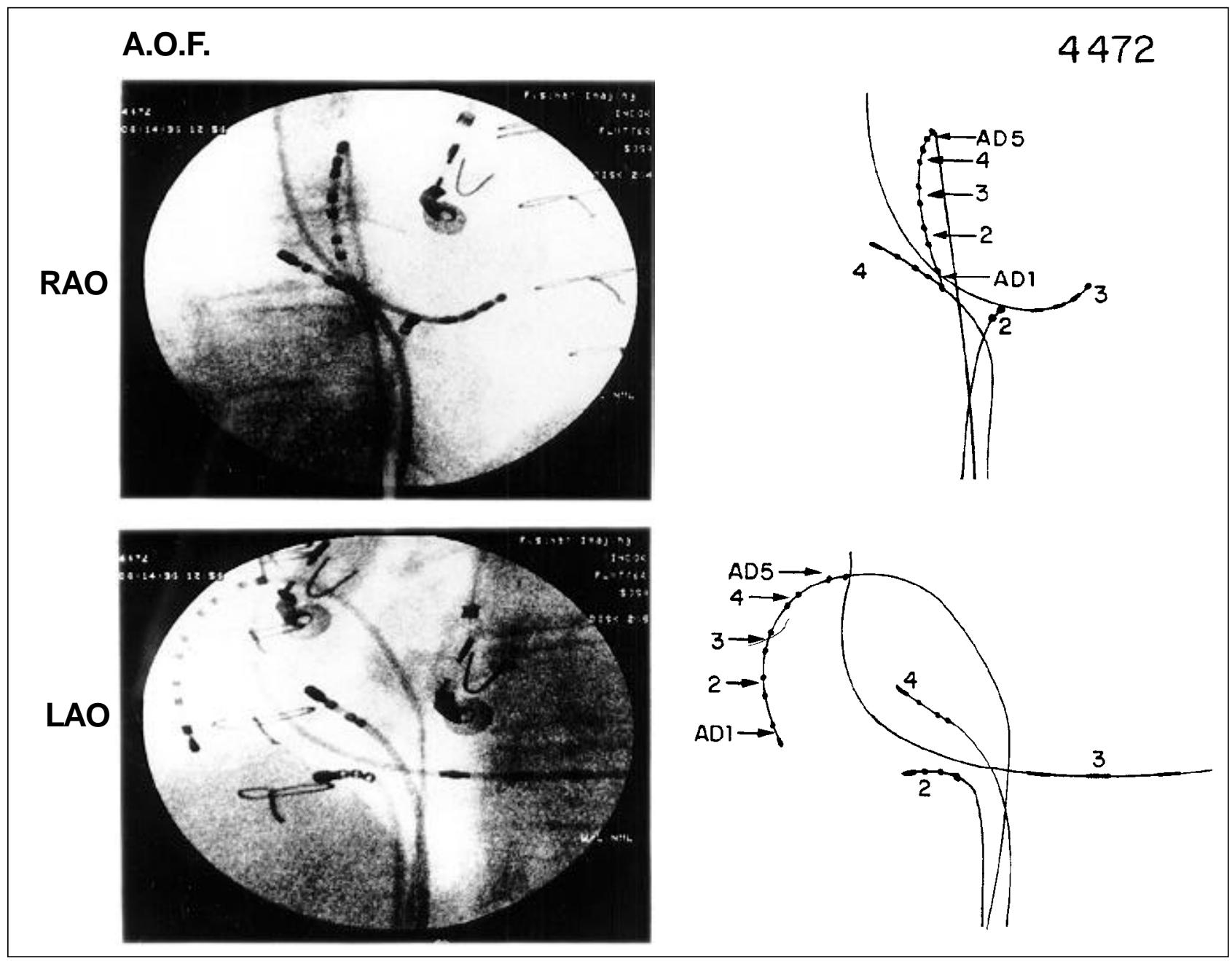

Fig. 3 - Ablação de FL em paciente do grupo II. Aspecto fluoroscópico dos cateteres nas projeções oblíqua anterior direita (superior) e oblíqua anterior esquerda (inferior). As setas apontam o cateter decapolar com cinco pares de eletrodos dispostos no sentido do par distal (AD = átrio direito distal) para o par proximal (AP = átrio direito proximal). ADD representa a entrada do istmo veia cava inferior (VCI) - anel da valva tricúspide (AT); 2 - é o par distal do cateter que explora o istmo VCI-AT; 3 - é o par proximal do cateter posicionado no seio coronário que representa a saída do istmo VCI-AT e 4 é o par distal do cateter posicionado na borda superior e posterior da VCI. 


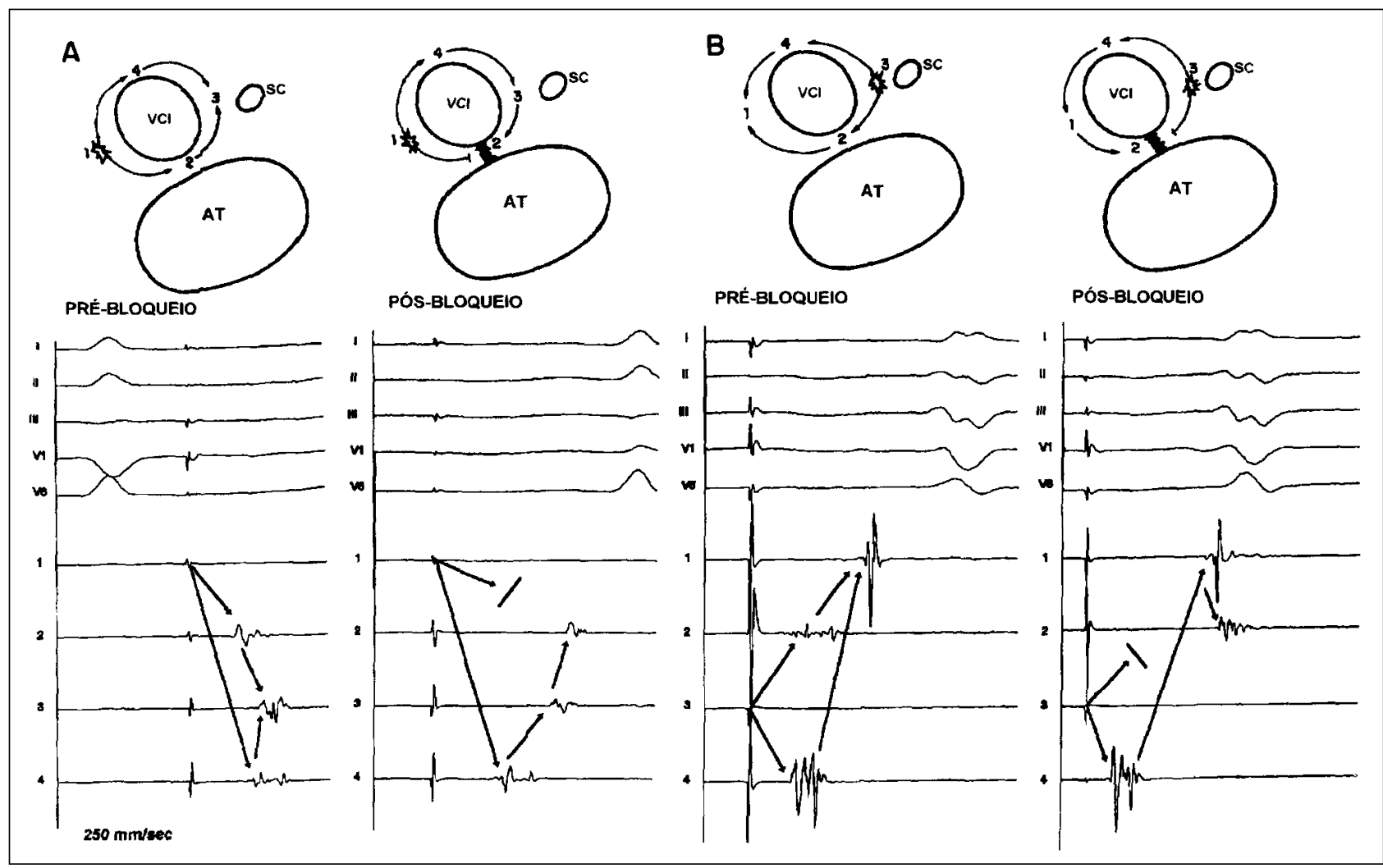

Fig. 4 - O painel A representa a avaliação da condução pelo istmo veia cava inferior (VCI) - anel da valva tricúspide (AT) no sentido anterógrado. Note a inversão da ativação em torno da VCI após o bloqueio do istmo VCI-AT. O painel B representa a avaliação da condução pelo istmo VCI-AT no sentido retrógrado; SC- óstio do seio coronário; 1 - entrada do istmo; 2 - istmo medial; 3 - saída do istmo; 4 - borda superior da VCI.

aplicações, tempo de procedimento, tempo de fluoroscopia, tempo de seguimento através do teste t de Student, para duas amostras independentes, quando obtido critério de normalidade na distribuição das variáveis. Caso contrário, foi usado o teste de Mann-Whitney. As proporções de sucesso, e ocorrência de FA nos GI e GII foram comparadas pelo teste exato de Fisher, e estabelecendo-se nível de significância estatística quando $\mathrm{p}<0,05$.

\section{Resultados}

Imediatos - Nos 30 pacientes iniciais (GI), 17 apresentavam ritmo de flutter tipo I no início do procedimento e 13, ritmo sinusal. Nestes últimos, flutter atrial sustentado do tipo I foi induzido com estimulação atrial rápida. Vinte e seis pacientes $(86,6 \%)$ atingiram os critérios de interrupção e não reindução do flutter, após uma média de 16,6 6 aplicações de RF, na $1^{\mathrm{a}}$ sessão em 25 (96\%) pacientes na $2^{\mathrm{a}}$ sessão em um (4\%), realizada na mesma internação (tab. II). A duração das sessões foi em média de $141 \pm 54$ min e a exposição média à fluoroscopia de $38,9 \pm 20$ min por paciente.

Nos 10 pacientes finais (GII), quatro apresentavam ritmo sinusal e seis apresentavam flutter atrial sustentado. As aplicações de RF foram realizadas durante flutter em oito casos e a monitorização da sequiência de ativação atrial demonstrou que o bloqueio ocorreu no istmo medial, caracterizado pela interrupção do flutter entre o eletrograma 1 (en-

\begin{tabular}{|llll|}
\hline \multicolumn{4}{|c|}{ Tabela II - Resultados imediatos da ablação do } \\
nos grupos I e II
\end{tabular}

trada do istmo) e 3 (saída do istmo). Todos atingiram os critérios de interrupção e não reindução de flutter atrial. Em dois pacientes, as aplicações não foram realizadas em ritmo de flutter. No $1^{\circ}$ não foi possível a indução flutter atrial sustentado e no $2^{\circ}$, a tentativa de indução de flutter provocou vários episódios de FA, revertidos com cardioversão elétrica. Nestes dois casos, procedeu-se às aplicações de RF durante estimulação do cateter 1, monitorizando-se a condução pelo istmo. Bloqueio bidirecional ao nível do istmo VCIAT foi demonstrado em 7 (70\%) pacientes e bloqueio unidirecional em três. Nestes últimos, o bloqueio ocorreu no sentido da entrada para a saída do istmo VCI-AT (sentido anti-horário). No total foram realizadas média de $11,6 \pm 6$ aplicações de RF. A duração média das sessões foi de $146 \pm 47$ min e exposição média à fluoroscopia foi de 
$31,9 \pm 15$ min por paciente. Os pacientes permaneceram internados por 24 a $48 \mathrm{~h}$ para observação clínica e não foram detectadas complicações nessa fase.

Seguimento - No GI, dos 26 pacientes com sucesso imediato, $23(88 \%)$ tiveram seguimento clínico. Após período médio de seguimento de $18 \pm 6$ meses, sete (30\%) pacientes apresentaram recorrência clínica de flutter e seis (26\%) evoluíram com episódios de FA. As recorrências de flutter ocorreram dentro dos primeiros seis meses de seguimento, exceto em um caso, que recorreu 11 meses após. Dos sete pacientes com recorrência, cinco apresentaram flutter isolado (quatro do tipo I e um do tipos I e II) e dois associados a crises esporádicas de FA. Em três casos foi realizado um segundo procedimento de ablação com sucesso; três controlados com drogas antiarrítmicas e um submetido à cirurgia do labirinto ${ }^{18}$. Dos seis pacientes com FA isolada, três foram controlados com drogas antiarrítmicas, dois submetidos à ablação da junção atrioventricular e implante de marcapasso definitivo e, em um, realizada cirurgia do labirinto.

No GII, um paciente com doença pulmonar obstrutiva crônica e miocardiopatia dilatada apresentou morte súbita 10 dias após a alta hospitalar, e nove pacientes tiveram seguimento tardio (média de $15 \pm 3 \mathrm{~m}$ ). Os seis pacientes com demonstração eletrofisiológica de bloqueio bidirecional não apresentaram recorrência clínica de flutter. Desses, dois voltaram a tomar amiodarona $(200 \mathrm{mg} / \mathrm{dia})$, por indicação de seus clínicos devido a extra-sístoles ventriculares sintomáticas (sem recorrência de flutter ou FA). Os três pacientes com bloqueio unidirecional apresentaram recorrência clínica de flutter $(\mathrm{p}=0,012)$. Um desses, com flutter tipo I, foi submetido a novo procedimento que resultou em bloqueio bidirecional e, após período de 11 meses de seguimento, não apresentou novos episódios de flutter. Os outros dois evoluíram com flutter tipo I, tipo II e episódios paroxísticos de FA, apesar do uso de amiodarona e foram submetidos à ablação da junção A-V, seguida de implante de marcapasso definitivo. Um paciente com flutter crônico, associado a miocardiopatia dilatada, apresentou episódio de acidente vascular cerebral isquêmico $36 \mathrm{~h}$ após o procedimento, evoluindo com seqüela motora. Na tabela III estão comparados os resultados obtidos com os pacientes dos GI e GII durante o seguimento clínico.

\begin{tabular}{llll|}
\hline \multicolumn{4}{c}{ Tabela III - Resultados observados durante o seguimento clínico nos } \\
grupos I e II
\end{tabular}

\section{Discussão}

Os resultados deste trabalho confirmam que a taxa de interrupção do flutter é alta, utilizando-se a técnica de bloqueio anatômico do istmo VCI-AT ${ }^{1-7}$ e que a recorrência clínica relaciona-se à ausência de bloqueio bidirecional ${ }^{13-17}$. Dos 40 pacientes estudados, em 36 (90\%) obtivemos sucesso imediato. Durante o seguimento clínico $30 \%$ apresentaram recorrência de flutter, não havendo diferença entre os pacientes de ambos os grupos (GI e GII). Entretanto, a análise retrospectiva dos pacientes do GII sugere fortemente, que a persistência de condução pelo istmo, mesmo que unidirecional, relaciona-se com recorrência clínica de flutter. A partir desses dados podemos inferir que em torno de $30 \%$ dos pacientes submetidos a ablação de flutter, guiada pelos critérios de interrupção e não reindução, podem apresentar recorrência clínica devido à ausência de bloqueio bidirecional na condução pelo istmo e que esta pode ser reconhecida no final do procedimento, através de uma avaliação eletrofisiológica simples.

Cosio e col ${ }^{19}$ já haviam observado que a simples interrupção do flutter, durante a aplicação de RF no istmo VCIAT, não poderia ser considerada critério de sucesso, já que $50 \%$ dos pacientes, quando submetidos a estimulação atrial imediata, apresentavam reindução, na maioria das vezes do tipo I e, em algumas, do tipo II. Salientaram a necessidade de estimulação atrial rigorosa como critério de sucesso. Durante um seguimento de até cinco anos, $46 \%$ dos pacientes apresentaram recorrência clínica, mais freqüente nos primeiros três meses e em pacientes com cardiopatias estruturais. A taxa de recorrência variou entre $15 \%$ e $35 \%$ na observação de vários autores ${ }^{1-7}$. Todos esses grupos tinham como critério de avaliação do procedimento a interrupção do flutter durante aplicação de RF e não reindução com estimulação atrial.

O fenômeno da recorrência do flutter, após a ablação com RF, pode ser explicado por três hipóteses: 1) a taxa de reprodução do flutter em laboratórioé relativamente menor do que a de outras taquicardias supraventriculares e, uma vez interrompido a sua não reindução, não implica necessariamente em tratamento definitivo; 2) a lesão miocárdica produzida pela RF pode ter um caráter transitório e, apesar de se obter uma supressão completa da condução na área crítica do circuito do flutter, pode haver recuperação da mesma, posteriormente; 3) a não recorrência depende de um bloqueio bidirecional irreversível na condução pelo istmo, podendo haver, entretanto, interrupção do flutter apenas com bloqueio unidirecional.

Neste trabalho, estudamos a terceira hipótese. Para ablação do tecido localizado no istmo VCI-AT são necessárias múltiplas aplicações na tentativa de se realizar uma linha de bloqueio transmural. O deslocamento do eletrodo explorador sobre uma superfície irregular pode deixar pequenas áreas de tecido íntegro entre uma lesão e outra, ou não atingir completamente a camada subepicárdica, produzindo uma lesão não transmural. Durante a fase aguda, as lesões podem ser suficientes para interromper o flutter, mas o circuito pode se reinstalar, posteriormente. 
A técnica de avaliação da condução pelo istmo VCIAT que elaboramos, baseou-se na hipótese de que uma vez realizada a linha de bloqueio no istmo, um estímulo liberado na zona de entrada tem que, necessariamente, contornar a VCI ou o AT, para atingir o óstio do seio coronário e entrar retrogradamente pelo istmo; da mesma forma, um estímulo liberado na região do óstio do seio coronário deve passar pela porção posterior da VCI ou ânulo tricúspide, antes de penetrar no istmo VCI-AT.

Através da análise da sequiência de ativação atrial no istmo VCI-AT foi possível diferenciar um bloqueio bidirecional de um unidirecional e de um distúrbio de condução intra-atrial. Durante a realização do protocolo, percebemos que a informação obtida com o cateter 4 (região posterior da VCI) era desnecessária e que a inversão da sequiência de condução pelo istmo VCI-AT pôde ser objetivamente avaliada apenas com os três cateteres posicionados na entrada, meio e saída do istmo.

A maior parte dos investigadores tem utilizado um cateter duodecapolar para analisar a seqüência de ativação pelo AT durante a estimulação da entrada e saída do istmo e determinar a presença de bloqueio bidirecional ${ }^{13-15}$. Para sua utilização, entretanto, é necessário que o polígrafo disponha de múltiplos canais intravitários simultâneos, não acessível a todos os laboratórios de eletrofisiologia. O cateter duodecapolar, apóia-se em toda a extensão do istmo, o que pode dificultar a manipulação do cateter de ablação nessa região. Com a metodologia apresentada neste trabalho, ou seja, estimulando ambos os lados da suposta linha de bloqueio com três referências, duas fixas e uma móvel, é possível avaliar a condução pelo istmo, com cateteres convencionais. Além disso, pudemos acompanhar a mudança na sequiência de ativação atrial na parede livre do anel tricúspide, com um cateter octapolar na posição de entrada (cateter 1), sem o inconveniente de avançar na região medial do istmo VCI-AT. Concluindo, os dados obtidos neste trabalho permitem afirmar que é possível avaliar a condução bidirecional pelo istmo VCI-AT sem a utilização de um cateter duodecapolar.

Embolia arterial pulmonar ou sistêmica após ablação com RF do flutternão foram descritas na literatura. Em nossa casuística, embolia arterial sistêmica ocorreu em um portador de miocardiopatia dilatada e flutter crônico. O procedimento transcorreu sem intercorrências, obtendo-se uma linha de bloqueio bidirecional no istmo VCI-AT; 36hs após o procedimento (após a alta hospitalar), o paciente apresentou embolia cerebral evoluindo com hemiparesia. Como esse paciente não apresentava comunicação interatrial, inferimos que a embolia fora devida à reversão do flutter atrial. Não se pode afastar a origem ventricular esquerda, principalmente por ser portador de cardiomiopatia dilatada, porém a relação temporal do evento, com a reversão do flutter para ritmo sinusal, sugere fortemente a câmara atrial esquerda como origem da embolia.

Não realizamos anticoagulação sistêmica ou ecocardiograma transesofágico previamente ao procedimento; nossa conduta baseou-se na observação de que a embolização pe- riférica é infreqüente após reversão elétrica do flutter ${ }^{20,21}$. Durante o seguimento, um outro paciente apresentou dispnéia súbita e morreu na unidade de emergência, 10 dias após o procedimento. Supôs-se fenômeno embólico no território pulmonar, entretanto o diagnóstico não foi confirmado por estudo anatomopatológico.

Embora não tenham relatado complicações embólicas durante ablação de flutter atrial, Cosio e col ${ }^{19}$ recomendaram o uso de anticoagulante oral nas duas semanas que antecedem o procedimento em pacientes com flutter crônico e manutenção de aspirina $250 \mathrm{mg} /$ dia pelas quatro a seis semanas seguintes. Nakagawa e col ${ }^{14}$ prescreveram $325 \mathrm{mg}$ de aspirina no dia anterior ao procedimento e mantiveram-na por seis semanas. Outros autores ${ }^{1,-3-7,15-17}$ não recomendam anticoagulação e não relataram complicações embólicas.

Frente às novas informações da possível trombogenicidade do flutter $^{22}$ e aos eventos observados na nossa casuística, passamos a admitir que pacientes com flutter crônico, principalmente quando associado à cardiopatia estrutural, devam ser submetidos a anticoagulação profilática para diminuir o risco de tromboembolismo. Entretanto, a resposta definitiva para esta conduta depende de trabalhos prospectivos, que possam responder especificamente a esta questão.

A FA é um evento freqüente após ablação do flutter atrial tipo I ${ }^{19,23}$. Sua causa não é conhecida. A hipótese mais aceita é que a fibrilação e oflutter atrial partilham uma disfunção atrial que dá origem aos circuitos reentrantes. Tem sido sugerido, como fatores preditivos para ocorrência de FA após a ablação do flutter, a sua ocorrência espontânea antes da ablação do flutter; sua indução após a ablação doflutter e presença de cardiopatia estrutural ${ }^{23}$.

Em nossa casuística, a incidência de FA foi de 25\%, mas não encontramos fatores preditores para sua ocorrência. Em ambos os grupos, não havia informação objetiva da presença de FA prévia à ablação do flutter. A presença de cardiopatia associada foi semelhante e não se observou diferença no número de aplicações de RF. No GI, três pacientes foram controlados com drogas antiarrítmicas, (dois, após indução de bloqueio atrioventricular total com implante de marcapasso definitivo) e um, submetido à cirurgia do labirinto. Os dois pacientes do GII foram submetidos à ablação da junção atrivoventricular eimplante de marcapasso definitivo.

Concluindo, este trabalho confirma observações anteriores que a aplicação linear de RF entre a VCI e o AT pode criar uma barreira à condução do estímulo e interromper o circuito do flutter. Entretanto, assumir que uma linha de bloqueio irreversível foi criada no istmo VCI-AT, baseandose na interrupção e a não reindução do flutter, após aplicação de RF, está relacionada com recorrências freqüentes durante o seguimento tardio. A condução pelo istmo VCI-AT pode ser avaliada, objetivamente, durante o procedimento e sua monitorização permite que as aplicações possam ser realizadas em ritmo sinusal, prescindindo da indução do flutter. A ausência de bloqueio bidirecional no istmo VCIAT correlaciona-se com recorrência tardia e, portanto, o objetivo final da ablação do flutter deve ser a demonstração de bloqueio bidirecional no istmo VCI-AT. 


\section{Referências}

1. Feld GK, Fleck RP, Chen PS et al - Radiofrequency catheter ablation for the treatment of human type I atrial flutter: identification of a critical zone in the reentrant circuit by endocardial mapping techniques. Circulation 1992; 86: 1233-40.

2. Cosio FG, Lopez GM, Goicolea A et al - Radiofrequency ablation of the inferior vena cava-tricuspide valve isthmus in common atrial flutter. Am J Cardiol 1993;71: 705-9.

3. Lesh MD, Van Hare GF, Epstein LM et al - Radiofrequency catheter ablation of atrial arrhythmias. Circulation 1994; 89: 1074-89.

4. Kirkorian G, Moncada E, Chevalier P et al - Radiofrequency ablation of atrial flutter. Circulation 1994; 90: 2804-14.

5. Calkins H, Leon AR, Deam AG et al - Catheter ablation of atrial flutter using radiofrequency energy. Am J Cardiol 1994; 73: 353-6.

6. Sosa EA, Scanavacca MI, Lewandowski A et al - Ablação com radiofrequiência do flutter atrial tipo I. Arq Bras Cardiol 1994; 63: 191-5.

7. Cruz FES, Fagundes MLA, Boghossian S et al - Terapia ablativa do flutter atrial tipo I com radiofrequiência. Arq Bras Cardiol 1995; 64: 323-30.

8. Klein G, Guiraudon G, Sharma A et al - Demonstration of macroreentry and feasibility of operative therapy in common type of atrial flutter. Am J Cardiol 1986; 57: 587-91.

9. Cosio FG, Arribas F, Palacios J et al - Fragmented electrograms and continous electrical activity in atrial flutter. Am J Cardiol 1986; 57: 1309-14.

10. Cosio FG, Arribas F, Barbero JM et al - Validation of double-spike electrograms as markers of conduction delay or block in atrial flutter. Am J Cardiol 1988; 61: 775-80.

11. Oshansky B, Okomura K, Hess PG et al - Demonstration of an area of slow conduction in human atrial flutter. J Am Coll Cardiol 1990; 16: 1639-48.

12. Cosio FG, Lopez GM, Goicolea A et al - Electrophysiologic studies in atrial flutter. Clin Cardiol 1992; 15: 667-73.
13. Poty H, Saoudi N, Abdel Aziz A et al - Radiofrequency catheter ablation of type I atrial flutter. Prediction of late success by electrophysiological criteria. Circulation 1995; 92: 1389-92.

14. Nakagawa H, Lazzara R, Khastgir T et al - Role of the tricuspid annulus and the Eusthachian valve/bridge on atrial flutter. Circulation 1996; 94: 407-24.

15. Cauchemez B, Haissaguerre M, Fisher B, Thomas O, Clementy J, Coumel P Electrophysiological effects of catheter ablation of inferior vena cava-tricuspid annulus isthmus in common atrial flutter. Circulation 1996; 93: 284-94.

16. Fisher $B$, Jais $P$, Shah $D$ et al - Radiofrequency catheter ablation of commom atrial flutter in 200 patients. J Cardiovasc Electrophysiol 1996; 7: 1225-33.

17. Chen SA, Chiang CE, Wu TJ et al - Radiofrequency catheter ablation of commom atrial flutter: Comparison of eletrophysiologically guided focal ablation technique and linear technique. J Am Coll Cardiol 1996; 27: 860-8

18. Cox JL, Boineau JP, Schuessler RB et al - Five-year experience with the Maze procedure for atrial fibrillation. Ann Thorac Surg 1993; 56: 814-24.

19. Cosio FG, Arribas F, Lopez-Gil M, Gonzalez HD - Radiofrequency ablation of atrial flutter. J Cardiovasc Electrophysiol 1996; 7: 60-70.

20. Arnold AZ, Mick MJ, Mazurek RP, Loop FD, Trohman RG - Role of prophylatic anticoagulation for direct current cardioversion in patients with atrial fibrillation or atrial flutter. J Am Coll Cardiol 1992; 19: 851-5.

21. Pagadala P, Gummadi SS, Olshansky B - Tromboembolic risk of chronic atrial flutter: is the risk underestimated? Circulation 1995; 90: I-398.

22. Irani WN, Grayburn PA, Afridi I - Prevalence of trombus, spontaneous echo contrast, and atrial stunning in patients undergoing cardioversion of atrial flutter. Circulation 1997; 95: 962-6.

23. Philippon F, Plumb VJ, Epstein AE et al - The risk of atrial fibrillation following radiofrequency catheter ablation of atrial flutter. Circulation 1995; 92: 430-5. 